\title{
Grundlagenkarten für die Raumplanung
}

\section{Einleitung}

Mit dem Bundesbeschluss über dringliche Massnahmen auf dem Gebiete der Raumplanung (1), der sich räumlich fast ausschliesslich auf ländliche Räume bezieht, hat die Bundesversammlung ihren Willen dokumentiert, die Schönheit unserer Landschaften und die Eigenart von Naturund Kulturdenkmälern zu erhalten sowie Erholungsund Gefahrengebiete zu sichern. In den Bestimmungen des Entwurfes zu einem neuen Raumplanungsgesetz (2) wurde der Grundsatz der Erhaltung bzw. der haushälterischen Nutzung der natuirlichen Lebensgrundlagen wie Boden, Luft, Wasser, Wald und Landschaft sowie die Gewährleistung einer ausreichenden eigenen Ernährungsbasis unseres Landes bestätigt und präzisiert. Der Wirkungsbereich der Raumplanung, die sich bisher schwergewichtig mit den Siedlungsgebieten befasst hatte, wird damit erweitert und bezieht ländliche Räume vermehrt mit ein.

Als ländliche Räume verstehen wir in diesem Zusammenhang in erster Linie Produktionsgebiete für die Land- und Forstwirtschaft (3). Daneben enthalten sie mit ihren teilweise noch ursprünglichen oder zumindest naturnahen Landschaften auch bedeutende Erholungs- und Schutzgebiete. $\mathrm{Zu}$ den ländlichen Räumen sind auch Landschaften zu rechnen, die gemeinhin noch den Charakter traditioneller Kulturlandschaften tragen, Gebiete also, deren Siedlungs- und Wirtschaftsstruktur formal von der Vergangenheit zeugen und mithin einen grossen Erholungswert aufweisen.

Dessen ungeachtet wird auch in Zukunft ein Teil der baulichen Entwicklung im sekundären und tertiären Wirtschaftssektor auf Kosten dieser ländlichen Räume vor sich gehen müssen. Wir denken in diesem Zusammenhang an die Erweiterung von Siedlungsgebieten in der Umgebung der Siedlungszentren, an die Umstrukturierung in verschiedenen Branchen der Wirtschaft, dann aber auch an Einrichtungen des Verkehrs, der Versorgung u.a.m.:

Die ländlichen Räume liegen in einem Spannungsfeld von gegensätzlichen Interessen.

Es ist daher ein wichtiges Anliegen der Raumplanung, auch für die Planung in ländlichen Räumen soweit.als möglich objektive Entscheidungsgrundlagen bereitzustellen. Das ist das
Ziel der hier vorzustellenden Karten. Selbstredend können diese Unterlagen ebenso gut für konzeptionelle Studien in urbanen Räumen angewendet werden - ihr Hauptanliegen ist es jedoch, die Datenlage in den ländlichen Gebieten unseres Landes zu verbessern. Konkret dienen die Kartenwerke nicht nur als Unterlagen für die Sachplanungen des Bundes; sie sind in erster Linie als Dienstleistungen zuhanden der kantonalen Planungsstellen zu verstehen. Sie vermitteln ihnen eine Reihe sachlich-objektiver Informationen insbesondere für die Erarbeitung von Richtplänen.

Bereits früher, anlässlich der Leitbildarbeiten des Institutes für Orts-, Regional- und Landesplanung ORL der ETH-Zürich, wurde eine erste Serie von Grundlagenkarten für die Raumplanung im Massstab 1: 300'000 erstellt. Diese erlaubten generelle Uebersichten über die naturräumlichen Voraussetzungen in der Schweiz, welche von der Raumplanung als Randbedingungen zu berücksichtigen sind. Schon bald aber zeigte es sich, dass die Karten im Massstab 1 :

300'000 verfeinert werden mussten, um sie für die Planungsarbeiten in Kantonen und Regionen wirklich anwenabar zu machen.

Die verschiedenen Untersuchungen wurden von einer interdepartementalen Arbeitsgruppe des Bundes unter der Leitung des Delegierten für Raumplanung begleitet. Die Ausführung der Studien lag bei Amtsstellen oder Forschungsanstalten der Eidgenossenschaft, zum Teil auch bei Universitätsinstituten oder privaten Vereinigungen, letztere im Auftrage von Bundesstellen.

In den folgenden Abschnitten werden die einzelnen Kartenwerke kurz skizziert und die Ergebnisse der Untersuchungen zusammengefasst. Auf die speziellen Probleme der Datenerfassung, Aufbereitung und deren kartographische Umsetzung kann in diesem Zusammenhang nicht eingegangen werden. Wir verweisen dazu auf die Berichte, die den Kartenwerken jeweils beigegeben sind. Die Publikationen sind, soweit nicht anders vermerkt, bei der Eidgenössischen Drucksachen und Materialzentrale EDMZ, 3003 Bern zu beziehen.

Dr. Heinrich Leuzinger, Mitarbeiter des Delegierten für Raumplanung, Bundesrain 20, 3003 Bern. 


\section{Grundlagenkarten für die Landwirtschaft}

2.1. Wärmegliederung der Schweiz aufgrund von phänologischen Geländeaufnahmen in den Jahren 1969 bis 1973

4 Karten 1:200'000

Beilage: Gebiete unterschiedlichen Föhneinflusses auf die phänologische Entwicklung der Pflanzendecke und die davon abgeleiteten Wärmestufen in der Schweiz

1 Karte 1 : 500'000

Leitung und Durchführung der Untersuchungen: Prof. Dr. K.F. Schreiber und Mitarbeiter.

Die vier Kartenblätter im Massstab 1:200'000 vermitteln gesamtschweizerisch ein Bild der Wärmegliederung in 19 Stufen auf der Grundlage von phänologischen Geländeaufnahmen in den Jahren 1969 bis 1973. Unter dem Vorbehalt weiterer Standorteinflüsse erlaubt die Wärmegliederung eine Interpretation für die Eignung der in der Legende aufgeführten landwirtschaftlichen Kulturen. Zum Vergleich der relativen Wärmegliederung mit den meteorologischen Messungen sind den Stufen entsprechende Klima-Kennziffern zugeordnet. Der Südföhn hatte in den einzelnen Kartierungsjahren zu einer unterschiedlichen Verfrühung der phänologischen Entwicklung geführt. Dieser Einfluss auf die Wärmeabstufung der vom Föhn bevorzugten Täler wurde in einer Kartenbeilage im Massstab 1:500'000 in 6 Stufen beriucksichtigt.

2.2. Klinaeignungskarten für die Landwirtschaft in der Schweiz

a) Klimaeignungskarte für die Landwirtschaft 4 Karten $1: 200 ' 000$

b) Karte der Klimaeignung für Getreide l Karte 1:500'000

c) Karte des Niederschlagshaushaltes 1 Karte 1:500'000

Bearbeitung: F. Jeanneret, Geographisches Institut der Universität Bern; P. Vautier, Station fédérale de recherches agronomiques, Changins sur Nyon

Leitung: Prof. Dr. B. Messerli, Dr. J. Caputa

Auf den vier Blättern der Karte 1:200'000 ist eine Gesamtbeurteilung der klimatischen Voraussetzungen und Einschränkungen für die Landwirtschaft, in 20 Kategorien, wiedergegeben.

Für eine solche Gesamtbeurteilung müssen die verschiedenen Kulturen in ein bestimmtes Wertverhältnis zueinander gesetzt werden. Deshalb erschien es nützlich, neben der Gesamteignung auch die für die Sicherung der ausreichenden Ernährungsbasis besonders wichtige Eignung für den Getreidebau in einer besonderen Karte darzustellen.

Als weiteres Ergebnis der Auswertung der Niederschlagsdaten fiel schliesslich eine Karte des Niederschlagshaushaltes an, welche sich, infolge der unterschiedlichen Sommerniederschläge, deutlich von den bekannten Niederschlagskarten auf der Basis der Jahresmittel abhebt. Da es sich um eine neuartige Karte handelt, wurde sie als Beilage in diese Arbeit aufgenommen.(4).

Die Karten können als Grundlage für gesamtschweizerische, kantonale oder regionale raumplanerische Untersuchungen dienen. Die Klimaeignungskarte 1: 200'000 will vor allem einen Ueberblick im gesamtschweizerischen Rahmen bieten. Auf der Stufe der Kantone, Regionen und Gemeinden können die Angaben aus dieser Untersuchung durch lokale Erhebungen ergänzt und damit der besonderen Problemlage angepasst werden.

\subsection{Bodeneignungskarte der Schweiz}

a) Bodeneignungskarte der Schweiz 4 Karten 1: 200'000, 1 Legende

b) Bodeneignungskarten 1:50'000 3 Karten 1 : 50'000: Genève, Solothurn, Zürich, 1 Legende

c) Ausschnitt aus einer Bodenkarte 1:25'000 1 Kartenausschnitt 1: 25'000 mit Legende

Bearbeitung: Eidgenössische Forschungsanstalt für landwirtschaftlichen Pflanzenbau Zürich Reckenholz

Prof. Dr. E. Frei und Mitarbeiter

Mitwirkung: Eidgenössische Anstalt für das

forstliche Versuchswesen Birmensdorf

Prof. Dr. F. Richard

Die Karte im Massstab 1: 200'000 zeigt geomorphologisch und bodenkundlich ausgeschiedene Einheiten, welche anschliessend nach ihren landwirtschaftlichen und forstlichen Nutzungsmöglichkeiten beurteilt sind. Diese Beurteilung erfolgt aufgrund der bodenkundlichen Eigenschaften der Kartierungseinheiten. Es werden damit standortgemässe Nutzungsmöglichkeiten unabhängig von der derzeitigen Bodennutzung dargestellt.

Da im Massstab 1: 200'000 Geländeteile von $4 \mathrm{~km} 2$ Fläche auf $1 \mathrm{~cm} 2$ der Karte reduziert werden, ist es verständlich, dass die Kartierungseinheiten nicht alle Einzelheiten aufdecken können. Wesentliche Bodenunterschiede mussten generalisiert werden. Für regionale und örtliche Planungsprobleme sind deshalb Boden- und Eignungskarten der Massstäbe 1 : 50'000 und 1 : 25'000 heranzuziehen. Diesem Bericht sind einige Beispiele solcher Karten beigefügt. Damit sollen die unterschiedlichen Aufgaben und Zweckbestimmungen der Bodenkarten verschiedener Massstäbe veranschaulicht werden.

2.4. Landwirtschaftliche Bodeneignungskarte der Schweiz

1 Karte 1 : 300'000, 1 Legendenblatt

Bearbeitung: Eidgenössische Forschungsanstalt für landwirtschaftlichen Pflanzenbau Prof.Dr.E.Frei und Mitarbeiter.

Diese Karte 1: 300'000 gehört noch in die Serie der Studien, die im Rahmen der Leitbildarbeiten des Instituts für Orts-, Regional- und Landesplanung an der ETH-Zürich erarbeitet wurden. 
Sie entstand im Jahre 1973 und wurde dann 1975 in der Reihe "Grundlagen für die Raumplanung" des Delegierten für Raumplanung veröffentlicht.

Die Karte und ihr separates Legendenblatt vermitteln ein einheitliches, gesamtschweizerisches Bild der landwirtschaftlichen Bodenqualitäten und ihrer Anbaueignung auf der Grundlage der Bodentypen und der nutzungsbeschränkenden Faktoren. Die Aussagen über die Anbaueignung beziehen sich nicht auf das Waldareal, das wegen des Uebersichtsmassstabes kartographisch nicht ausgewiesen werden konnte.

Die Bodeneignungskarte 1:300'000 kann als Grundlage für nationale und kantonale Leitbildarbeiten und Konzepte dienen. Der Uebersichtsmassstab schliesst jedoch eine regionale oder gar lokale Anwendung aus. Dafür sind die Bodeneignungskarten in den Massstäben 1: 200'000 bis 1:25'000 notwendig. Der Planungs-, Landwirtschafts- und Forstfachmann wird daher zum unter Punkt 2.3. vorgestellten Bericht mit den detaillierten Kartenwerken greifen. Wer dagegen mehr Wert auf Uebersichtlichkeit legt, wird besonders für Unterrichtszwecke weiterhin die Bodeneignungskarte im Massstab 1 : 300 ' 000 benützen.

\section{Grundlagenkarten für die Forstwirtschaft}

\subsection{Gefahrenkarte der Schweiz}

22 Karten 1: 100'000

Bearbeitung: Eidgenössisches Oberforstinspektorat

Aufgrund des Bundesbeschlusses über dringliche Massnahmen auf dem Gebiete der Raumplanung vom 17. März 1972 haben die Kantone u.a. diejenigen Gebiete bezeichnet, welche durch Naturgefahren bedroht sind. Eine erste Sichtung dieser Ausscheidungen durch den Delegierten für Raumplanung und das Eidgenössische Oberforstinspektorat zeigte eine grosse Heterogenität in den Gebietsbezeichnungen. Dies veranlasste den Bund, zusammen mit den Kantonen eine einheitliche Karte der Gefahrengebiete im Massstab 1 : 100'000 zu erstellen.

Die Karte dient als Uebersicht über die von Naturgefahren bedrohten Gebiete der Schweiz. Sie ist als Grundlage für überregionale und gesamtschweizerische Arbeiten sowie als Ausgangspunkt für weitergehende Untersuchungen der Kantone und Gemeinden zu verwenden. Gemäss dieser Zielsetzung wurde das Hauptaugenmerk auf eine möglichst zuverlässige Abklärung der sicheren Gebiete gelegt. Für die verbindliche Beurteilung von Einzelfällen wird auf detaillierte Gefahrenzonenpläne in den Kantonen und Gemeinden verwiesen.

Alle Gebiete ohne Ueberdruck wurden bezüglich Lawinen, Steinschlag, Rutschungen (ausgenommen Ueberschwemmungen und Murgänge) als sicher beurteilt. Ein Restrisiko ist jedoch nicht überall auszuschliessen, schon weil Gefahrengebiete unter 1 ha auf einer Karte 1: 100'000 nicht darstellbar sind.

Bekannte Gefahrengebiete sind dunkelrot, nicht näher beurteilte oder potentiell gefährdete Flächen hellrot uiberdruckt. Hellrote Signatur kann deshalb sowohl eine nicht abschliessende Beurteilung als auch ein verminderter Gefährdungsgrad bedeuten. Das erste gilt vor allem für die grossen hellroten Flächen im Alpengebiet.

Die Karte hat keine Rechtswirkung; es kann auch keine Haftung für die darin enthaltenen Angaben übernommen werden.

3.2. Brachlandproblem - Modelluntersuchungen und Uebersichtskarten $1: 300^{\prime} 000$

Die Karte befindet sich noch in Bearbeitung.

Bearbeitung: Eidgenössische Anstalt für das

forstliche Versuchswesen Birmensdorf

E. Surber

4. Grundlagenkarten für Fremdenverkehr und Erholung

4.1. Karte der Touristischen Transportanlagen der Schweiz

1 Karte $1: 300^{\prime} 000$

Das Missverhältnis zwischen der breitgefächerten Bedeutung Touristischer Transportanlagen einerseits und dem unvollständigen Wissen über die Anzahl, die Verteilung und die Leistungsfähigkeit aller dieser Anlagen andererseits gaben den Anlass zur Erarbeitung einer Karte über die Touristischen Transportanlagen der Schweiz sowie eines ergänzenden Berichtes, welcher detailliert Auskunft gibt über jede der aufgenommenen Anlagen.

Als touristische Transportanlagen werden alle Seilbahnen, Zahnradbahnen, Standseilbahnen, Schmalspurbahnen und Skilifte bezeichnet, welche ausschliesslich oder vorwiegend von Touristen benutzt werden. Bisher fehlte in unserem Land eine vollständige Uebersicht über alle bestehenden Anlagen dieser Art. Wohl gibt das Eidgenössische Amt für Verkehr seit 1948 eine Zusammenstellung aller eidgenössisch konzessionierten Anlagen heraus, uiber die Anlagen mit kantonaler Bewilligung - Skilifte und touristische Kleinluftseilbahnen - waren jedoch bis heute keine gesamtschweizerischen Unterlagen zu erhalten. Diese Lücke fiel namentlich für raumplanerische Arbeiten stark ins Gewicht, denn die kantonal bewilligten touristischen Transportanlagen haben heute einen Anteil von über $70 \%$ am gesamtschweizerischen Anlagebestand erreicht.

Die Aufarbeitung des Datenmaterials über touristische Transportanlagen verbessert die Beurteilungsgrundlagen, insbesondere bei der Bewilligung bzw. Konzessionierung neuer touristischer Transportanlagen. Im Vordergrund steht aber auch die Bereitstellung von Unterlagen für die Erarbeitung von touristischen Ausbau- und Entwicklungsvorstellungen im Rahmen der Raumplanungsverfahren von Gemeinden, Regionen und Kantonen. 


\subsection{Pflanzensoziologisch-ökologische Grundlagen-} karte

Die Karte befindet sich noch in Bearbeitung. Bearbeitung: Schweizerische pflanzengeographische Kommission (4).

Prof. Dr. H. Zoller

5.2. Inventar der Naturschutzgebiete und Naturdenkmäler der Schweiz

Landeskarten 1 : 25'000 mit Aufdruck

Bearbeitung: Schweizerischer Bund für Naturschutz

Das Inventar der Naturschutzgebiete und Naturdenkmäler der Schweiz enthält Gebiete und Objekte, welche ganz oder teilweise geschuitzt sind oder vom SBN als schuitzenswert erachtet werden.

Das Inventar liegt bei den zuständigen Amtsstellen sowie beim Bund für Naturschutz zur Einsicht auf. Eine Publikation erfolgt nicht.

\section{Anmerkungen}

(1) Bundesbeschluss über dringliche Massnahmen auf dem Gebiete der Raumplanung, vom 17. März 1972. Die befristeten Massnahmen wurden - nach der Ablehnung des Bundesgesetzes über die Raumplanung im Juni 1976 durch Volk und Stände - längstens bis zum 31. Dezember 1979 verlängert (Bundesbeschluss über die befristete Verlängerung von Massnahmen auf dem Gebiete der Raumplanung vom 8. Oktober 1976).

(2) Bundesgesetz über die Raumplanung, Entwurf Juni 1977. Der Entwurf zum neuen Raumplanungsgesetz wurde gleichzeitig mit der Eröffnung der Vernehmlassung im Heft 2/77 der "Raumplanung Schweiz", Informationsheft des Delegierten für Raumplanung veröffentlicht.

(3) Zur Problematik des Begriffes "ländlicher Raum" siehe: "Der ländliche Raum - eine Aufgabe der Raumplanung"; Festschrift für Theo Weidmann und Ernst Winkler, insbesondere: Elsasser H.; Der Ländliche Raum: Begriff und Abgrenzung in der Geographie, Heft 28 der Schriftenreihe zur Orts-, Regional- und Landesplanung; ORL-Institut der ETH Zürich.

(4) Vergleiche dazu die ausführlichen Artikel von $F$. Jeanneret $u$. 0 . Hegg in diesem Heft, S. 49-54 u. S. $45-48$.
LENDI Martin (Ed.), Der ländliche Raum - eine Aufgabe der Raumplanung, Festschrift für Theo Weidmann und Ernst Winkler, Schriftenreihe zur Orts-, Regional- u. Landesplanung, Nr.28, verschiedene Beiträge, 198 S., ORL-Institut, ETH Zürich 1977.

Im Jahre 1977 sind die beiden Professoren Theo Weidmann und Ernst Winkler von der Leitung des ORL-Institutes zurückgetreten. Zu Ehren dieser verdienten Wissenschafter gab $M$. Lendi die vorliegende Festschrift heraus. Auf 200 Seiten wirdigen 22 namhafte Autoren aus dem In- und Ausland die Stellung der Kulturtechnik und der Geographie innerhalb der Raumforschung, beschäftigen sich sodann eingehend mit Fragen ländlicher Räume - einem der wichtigsten Schaffensbereiche der Geehrten; der Schlussabschnitt ist internationalen Aspekten der Raumplanung gewidmet. Verschiedene Artikel umreissen m.E. klar die Fragen, welche gegenwärtig die Raumplanung in der Schweiz bewegen. So muss $M$. Lendi "eine Ueberbetonung der Methoden der planerischen Vorgehensweisen und der Entscheidfindung feststellen,(...) während (die raumplanerische Praxis) gleichzeitig in der Bearbeitung der materiellen Fragestellungen weitgehend im Stiche gelassen" werde (S.8). J. Maurer erwartet in diesem Zusammenhang von der Geographie wichtige Beiträge, denn sie sei in der Lage, "gleichsam als interdisziplinäre Disziplin der Raumforschung die zahlreichen Ëinzelerkenntnisse vieler anderer Fachgebiete über den Raum sinnvoll zusammenzufügen. (...) Geographie als Raumforschung und Raumplanung als Form der Entscheidungslogik könnten sich dann gegenseitig ergänzen und befruchten"(S.46).

Die Beiträge zum Thema "Ländlicher Raum" zeigen, dass in der Schweiz klare Planungsvorstellungen für diese - meist negativ als 'Nicht-Baugebiet' oder 'Uebriges Gemeindegebiet' bezeichneten Gebiete erst ansatzweise vorhanden sind. Zu lange standen in der Raumplanung einseitig Fragen der baulichen Nutzung im Vordergrund. Nicht umsonst erhielt der zweite Hauptabschnitt der Festschrift den Titel: "Zuwendung zu einer grossen Aufgabe: Der ländliche Raum".

Es ist zu hoffen, dass die Festschrift für Theo Weidmann und Ernst Winkler den angesprochenen Wissenschaften, namentlich der Geographie, richtungsweisende Impulse verleiht. Die Forschungsarbeiten der Geehrten bilden dazu ein tragfähiges Fundament - darauf gilt es aufzubauen :

GH 1/1978

Heinrich Leuzinger 